\title{
Effects of I6-week high-intensity interval
using upper and lower body ergometers on aerobic fitness and morphological changes in healthy men: a preliminary study
}

\author{
This article was published in the following Dove Press journal: \\ Open Access Journal of Sports Medicine \\ 4 November 2014 \\ Number of times this article has been viewed
}

\author{
Yusuke Osawa ${ }^{1,2, *}$ \\ Koichiro Azuma ${ }^{3, *}$ \\ Shogo Tabata ${ }^{3}$ \\ Fuminori Katsukawa ${ }^{2}$ \\ Hiroyuki Ishida ${ }^{2}$ \\ Yuko Oguma ${ }^{2}$ \\ Toshihide Kawai ${ }^{4}$ \\ Hiroshi Itoh ${ }^{4}$ \\ Shigeo Okuda ${ }^{5}$ \\ Hideo Matsumoto ${ }^{3}$ \\ 'Japan Society for the Promotion of \\ Science, Graduate School of Arts and \\ Sciences, University of Tokyo, Tokyo, \\ Japan; ${ }^{2}$ Sports Medicine Research \\ Center, Keio University, Kanagawa, \\ Japan; ${ }^{3}$ Institute for Integrated Sports \\ Medicine, School of Medicine, Keio \\ University, Tokyo, Japan; ${ }^{4}$ Department \\ of Internal Medicine, School of \\ Medicine, Keio University, Tokyo, \\ Japan; ${ }^{5}$ Department of Radiology, \\ School of Medicine, Keio University, \\ Tokyo, Japan \\ *Yusuke Osawa and Koichiro Azuma \\ are co-first authors of this article
}

Correspondence: Yusuke Osawa Japan Society for the Promotion of Science, Graduate School of Arts and Sciences, University of Tokyo, 3-8-I Komaba, Meguro-ku, Tokyo 153-8902, Japan

Tel +8I 354657646

Fax +8I 354546870

Email cosawa@mail.ecc.u-tokyo.ac.jp
Abstract: It is unclear whether combined leg and arm high-intensity interval training (HIIT) improves fitness and morphological characteristics equal to those of leg-based HIIT programs. The aim of this study was to compare the effects of HIIT using leg-cycling (LC) and arm-cranking (AC) ergometers with an HIIT program using only LC. Effects on aerobic capacity and skeletal muscle were analyzed. Twelve healthy male subjects were assigned into two groups. One performed LC-HIIT $(n=7)$ and the other LC- and AC-HIIT $(n=5)$ twice weekly for 16 weeks. The training programs consisted of eight to 12 sets of $>90 \% \mathrm{VO}_{2}$ (the oxygen uptake that can be utilized in one minute) peak for 60 seconds with a 60 -second active rest period. $\mathrm{VO}_{2}$ peak, watt peak, and heart rate were measured during an LC incremental exercise test. The cross-sectional area (CSA) of trunk and thigh muscles as well as bone-free lean body mass were measured using magnetic resonance imaging and dual-energy X-ray absorptiometry. The watt peak increased from baseline in both the LC $(23 \% \pm 38 \% ; P<0.05)$ and the LC-AC groups $(11 \% \pm 9 \% ; P<0.05)$. The CSA of the quadriceps femoris muscles also increased from baseline in both the LC $(11 \% \pm 4 \%$; $P<0.05)$ and the LC-AC groups $(5 \% \pm 5 \% ; P<0.05)$. In contrast, increases were observed in the CSA of musculus psoas major $(9 \% \pm 11 \%)$ and musculus anterolateral abdominal $(7 \% \pm 4 \%)$ only in the LC-AC group. These results suggest that a combined LC- and AC-HIIT program improves aerobic capacity and muscle hypertrophy in both leg and trunk muscles.

Keywords: arm-cranking ergometer, cycling ergometer, aerobic capacity, skeletal muscle

\section{Introduction}

High-intensity interval training (HIIT) is frequently used not only by athletes but also by untrained individuals and metabolic syndrome, type 2 diabetes, and cardiac patients as a safe and effective alternative to conventional endurance exercises. ${ }^{1-6}$. Although HIIT improves oxidative capacity, resting glycogen content, and peripheral vascular structure and function, ${ }^{7-10}$ HIIT programs can induce a greater feeling of fatigue as well as higher ratings of perceived exertion (RPE) compared with the classical moderateintensity continuous training. ${ }^{11,12}$ Thus, it may not be possible for untrained individuals or those with chronic diseases to perform long-term HIIT, though among recreationally active men it has been reported that HIIT was perceived to be more enjoyable, despite higher RPE than moderate-intensity continuous training. ${ }^{11}$

Though traditional HIIT employing the Wingate protocol results in significant physiological adaptations, it is extremely demanding and potentially unsafe for sedentary individuals. Therefore, several practical protocols were developed by decreasing 
the exercise intensity. ${ }^{13,14}$ Another possible approach for decreasing physical demand of the specific part of the body is to divide the HIIT exercise between the lower and upper limbs, instead of using only one body part. We speculated that the effects of HIIT exercise would also benefit areas of the body other than those directly involved in the exercise, as was previously demonstrated with other types of exercise, such as cross-training. ${ }^{15,16}$ However, it is unclear whether a combined leg and arm HIIT program would provide fitness benefits equal to those of an HIIT program of equal training intensity and training volume.

A number of studies have investigated the effects of shortterm HIIT and/or combined exercise on skeletal muscles. For example, 6- and 8-week HIIT programs did not induce muscle hypertrophy in athletes. ${ }^{17,18}$ In contrast, it was reported that an 8-week exercise program consisting of HIIT and continuous aerobic exercises led to a $24 \%$ increase in the cross-sectional area (CSA) of thigh muscles in middle-aged type 2 diabetic patients. ${ }^{19}$ Since local leg muscle aerobic capacity seems to influence the extent of muscle adaptations following endurance training, ${ }^{20}$ previous training status may explain the difference in these results. Moreover, in the early phase of resistance training, neural adaptation precedes muscular hypertrophy, ${ }^{21}$ and there seems to be a muscle-specific time course for muscular hypertrophy following resistance training. In fact, 8 weeks of training may not be enough for muscular hypertrophy in quadriceps muscles. ${ }^{22}$ Therefore, further evaluation of the effects of longer-term HIIT programs on skeletal muscle is warranted in nonathlete men.

We hypothesized that an HIIT program using a legcycling (LC) and an arm-cranking (AC) ergometer would improve aerobic fitness and morphological changes to the same extent as an HIIT program using an LC ergometer only. We therefore investigated the effects of a 16-week combined LC- and AC-HIIT program on aerobic fitness and muscle hypertrophy compared with an LC-HIIT program of similar duration and intensity in healthy adult males.

\section{Methods}

\section{Participants}

Fourteen healthy men (aged 28-48 years) volunteered for this study. Participants were recruited using posted advertisements. Only two participants engaged in regular exercise (soccer and gym-based training, respectively) once or twice a week. The eligibility criteria for participants were age 20-50 years and male. A physician evaluated the health and medical history, current medical conditions, and signs and risk factors of cardiovascular and orthopedic diseases of each potential participant. Participants were randomly assigned to groups that performed either an LC-HIIT program (LC group, $\mathrm{n}=8$ ) or a combined LC-AC-HIIT program (LC-AC group, $\mathrm{n}=6$ ). The study was approved by the local ethics committee of Keio University, and written informed consent was obtained from all participants.

\section{Procedures}

\section{Randomization procedure}

The participants were stratified by age and randomly assigned into two groups: an LC-HIIT program (LC group, $\mathrm{n}=8$ ) or a combined LC-AC-HIIT program (LC-AC group, $\mathrm{n}=6$ ). Figure 1 shows the flow of participants through the study.

\section{HIIT training program}

Participants performed the HIIT training program twice a week, consisting of 16- to 24-minute exercise sessions that were separated by at least 2 days. Although no studies have configured an HIIT program using both AC and LC ergometers, the program of exercise intensity, volume, and duration was based on interventions by Little et al..$^{23}$ The number of the repetitions was gradually increased from eight to 12 sets during the first 4 weeks, and after 4 weeks the number of the repetitions was adjusted depending on the participants' physical condition and was a minimum of eight sets, while the workload was progressively increased. After performing a standardized 10-minute warm-up involving body weight exercises (eg, knee-ups and hand-knee exercises), participants performed LC-HIIT on an electronically braked LC ergometer (Power Max VIII; Konami Sports \& Life Co, Ltd, Tokyo, Japan). The LC-HIIT consisted of ergometer exercise for 60 seconds at a workload of $>90 \% \mathrm{VO}_{2}$ peak, as determined from the results of an LC incremental exercise test that was conducted prior to the commencement of the training program. The pedaling rate was maintained at $>60 \mathrm{rpm}$ throughout the 60 -second HIIT exercise. Participants then pedaled at a rate equivalent to $30 \mathrm{~W}$ for 60 seconds during the recovery period. This was repeated for eight to 12 sets in the LC group and four to six sets in the $\mathrm{LC}-\mathrm{AC}$ group during each training session.

After completing the four to six sets of LC-HIIT, participants in the LC-AC group then immediately performed AC-HIIT on an electronically braked AC ergometer (Exite Top; Technogym, Tokyo, Japan). The AC-HIIT consisted of 60 -second ergometer exercise at a workload $>90 \%$ of peak workload, which was determined from an $\mathrm{AC}$ incremental exercise test, interspersed with a 60 -second active recovery period. During the active recovery period, participants rotated 


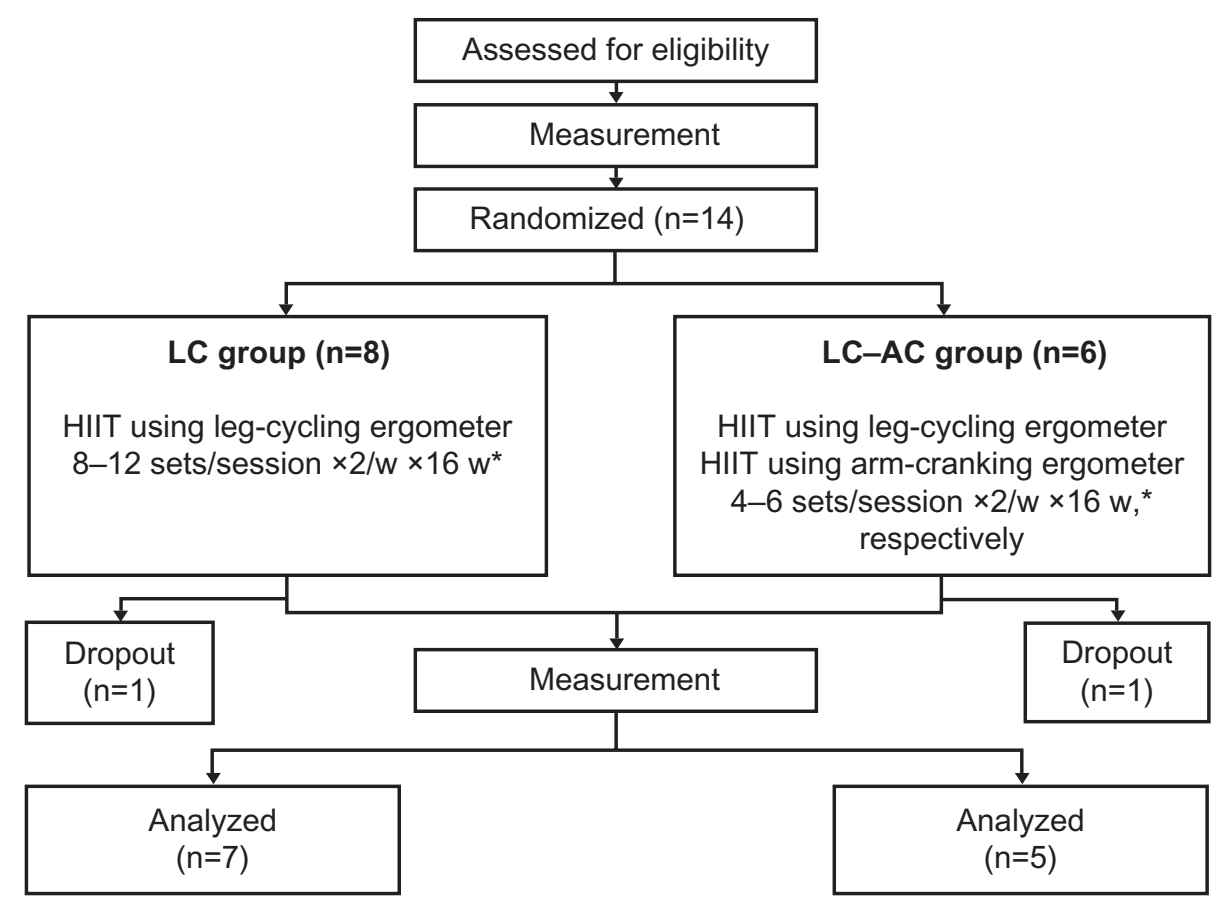

Figure I Flow of participants through the trial. Participants were randomly assigned to training groups that performed twice-weekly high-intensity interval training (HIIT) using either leg-cycling ergometers (LC group) or both LC and arm-cranking ergometers (LC-AC group) for 16 weeks.

Note: *twice weekly for 16 weeks.

the crank at $40 \mathrm{~W}$. Even if the relative exercise intensity in $\mathrm{AC}$ exercise was the same as that in LC exercise, the absolute exercise intensity in $\mathrm{AC}$ exercise was less than that in LC exercise. Thus, to minimize the difference in total work per session between the two groups, we configured greater workload during the active recovery period in AC-HIIT than that in LC-HIIT. In a pilot study, $40 \mathrm{~W}$ was the near maximal workload, which all participants could easily perform as the active recovery period. This was repeated for four to six sets in each session. To confirm the adequacy of the training intensity, blood lactate level was checked several times using Lactate Pro (Arkray, Kyoto, Japan).

Each training session, including the 10-minute warm-up and 5-minute cool-down exercises, lasted about 30-40 minutes in both groups. The adequacy of training intensity was evaluated using the Borg 6-20 scale, ${ }^{24}$ which was measured at the end of the sixth and last sets at every training session. All training sessions were supervised by a physician (KA) or a certified strength and conditioning specialist of the National Strength and Conditioning Association, Japan (YO).

\section{Measurements}

\section{Exercise testing}

Before and after the 16-week training period, in a physiciansupervised LC incremental exercise test, participants began cycling at $30 \mathrm{~W}$ and the power output was then increased by $15 \mathrm{~W}$ every minute until participants could no longer maintain the required pedaling rate. Expired gas was measured throughout the test using a pulmonary gas exchange system (Vmax-spectra; Sensor Medics, Yorba Linda, CA, USA). Electrocardiogram measurements were also recorded at the same time as the $\mathrm{VO}_{2}$ and $\mathrm{VCO}_{2}$ measurements using Cardiofax V (Nihon Koden, Tokyo, Japan). Before each test, the turbine of the Vmax system was calibrated with a 3-L syringe, and the Vmax system was also calibrated, with two different gases ( $26 \%$ oxygen, $0 \%$ carbon dioxide; and $16 \%$ oxygen, $4 \%$ carbon dioxide). Peak $\mathrm{VO}_{2}, \mathrm{~W}$, and heart rate (HR) were measured and used for further analyses. An AC incremental exercise test was also performed, starting at $40 \mathrm{~W}$ with increases by $10 \mathrm{~W}$ every 2 minutes, using a protocol by a recent report. ${ }^{25}$ It was technically difficult to monitor expired gas during AC. Therefore, only W and HR were monitored.

\section{Magnetic resonance imaging}

Magnetic resonance imaging (MRI) scans of trunk and thigh muscles were performed using a $1.5 \mathrm{~T}$ imager (Signa Excite HD Twin Speed; GE Healthcare, Waukesha, WI, USA). A set of T2-weighted single-shot fast spin echo images (TR/TE [reaction time/echo time] $=\infty / 90 \mathrm{~ms}$, FOV [field of view] $=38 \mathrm{~cm}$, slice thickness $=8 \mathrm{~mm}$ with $3 \mathrm{~mm}$ interval, matrix size $=288 \times 192$ ) was obtained for the abdomen 
centered at the L3-L4 disc space level. The CSAs of musculus (m) psoas major, $\mathrm{m}$ rectus abdominis, spinal muscle, and $\mathrm{m}$ anterolateral abdominal (obliquus internus abdominis, obliquus externus abdominis, and transversus abdominis) were determined from a single-shot fast spin echo image. Subsequently, a set of T2-weighted fast spin echo images $(\mathrm{TR} / \mathrm{TE}=4,000 / 90 \mathrm{~ms}, \mathrm{FOV}=30 \mathrm{~cm}$, slice thickness $=8 \mathrm{~mm}$ with $3 \mathrm{~mm}$ interval, matrix size $=320 \times 224$ ) taken at the halfway point of the femur (between the superior border of the patella and the greater trochanter) was used to measure the CSA of the quadriceps femoris and hamstring muscle. The obtained Digital Imaging and COmmunication in Medicine (DICOM) images were analyzed with Slice-O-Matic software (Tomovision, Quebec, Canada). The average CSA of the left and right muscles was used for further statistical analysis.

\section{Dual-energy X-ray absorptiometry}

Total and regional (upper body, trunk, and legs) bone-free lean body mass (LBM) and percentage of body fat were measured by whole-body dual-energy X-ray absorptiometry (DXA) (Lunar Prodigy Advance; GE Healthcare, Waukesha) scans using enCORE software version 9.2 performed at Keio University Hospital. Standard scan mode was used for the whole-body scans.

\section{Statistical analyses}

Intragroup differences in age, body mass, and percentage of body fat were evaluated by the Wilcoxon $t$-test. Percent changes from baseline were compared between groups using the MannWhitney $U$ test. A selective bivariate relationship (ie, watt peak (peak W) and $\mathrm{VO}_{2}$ peak in the incremental exercise test and percent change of the CSA of muscles) was investigated using Spearman's rank correlation coefficient. PASW software version 19.0 for Macintosh (IBM Japan, Tokyo, Japan) was used for all statistical analyses. The level of significance was set at $P<0.05$, and all values are presented as mean \pm standard deviation. Since this is a pilot study, we did not calculate sample size.

\section{Results}

\section{Baseline characteristics}

The characteristics of the participants are summarized in Table 1. No differences between the LC and LC-AC groups at baseline were observed in any of the evaluated characteristics. Two participants dropped out of the study due to work-related conflicts ( $\mathrm{n}=1, \mathrm{LC}$ group) and aggravated lower-back pain ( $\mathrm{n}=1, \mathrm{LC}-\mathrm{AC}$ group), which did not occur during a training session. The remaining participants $(n=12)$ completed the 16-week HIIT programs (Figure 1).
Table I The characteristics of the participants in the present study

\begin{tabular}{llllll}
\hline Participant & $\begin{array}{l}\text { Age } \\
\text { (years) }\end{array}$ & $\begin{array}{l}\text { BW } \\
(\mathbf{k g})\end{array}$ & $\begin{array}{l}\text { BMI } \\
\left(\mathbf{k g} \cdot \mathbf{m}^{-2}\right)\end{array}$ & $\begin{array}{l}\mathbf{V O}_{2} \text { peak } \\
\left(\mathbf{m L} \cdot \mathbf{m i n}^{-1} \cdot \mathbf{k g}^{-1}\right)\end{array}$ & $\begin{array}{l}\text { Body fat } \\
(\%)\end{array}$ \\
\hline LC & & & & & \\
LI & 34 & 81.2 & 27.0 & 37.9 & 25.6 \\
L2 & 33 & 71.2 & 24.9 & 33.0 & 29.3 \\
L3 & 28 & 60.4 & 20.0 & 41.6 & 17.0 \\
L4 & 29 & 77.0 & 25.0 & 39.5 & 25.0 \\
L5 & 41 & 76.0 & 24.5 & 35.8 & 24.1 \\
L6 & 36 & 67.9 & 24.8 & 48.0 & 21.0 \\
L7 & 34 & 71.8 & 22.3 & 52.0 & 13.2 \\
Mean & 33.6 & 72.2 & 24.1 & 41.1 & 22.2 \\
SD & 4.0 & 6.3 & 2.1 & 6.2 & 5.5 \\
LC-AC & & & & & \\
A1 & 29 & 75.4 & 23.5 & 44.9 & 18.9 \\
A2 & 28 & 87.6 & 29.6 & 40.6 & 29.0 \\
A3 & 42 & 76.5 & 23.6 & 38.0 & 24.6 \\
A4 & 48 & 71.5 & 23.4 & 31.4 & 22.1 \\
A5 & 38 & 75.5 & 27.1 & 37.2 & 33.0 \\
Mean & 37.0 & 77.3 & 25.4 & 38.4 & 25.5 \\
SD & 7.6 & 5.4 & 2.5 & 4.4 & 5.6 \\
\hline
\end{tabular}

Abbreviations: BMI, body mass index; BW, body weight; LC, leg-cycling group; LC-AC, leg-cycling and arm-cranking group; SD, standard deviation; $\mathrm{VO}_{2}$, oxygen uptake that can be utilized in one minute.

\section{RPE during HIIT}

While absolute workload was about $40 \%$ for AC vs LC (117 W vs equivalent of $282 \mathrm{~W}$ ), RPE after AC was similar

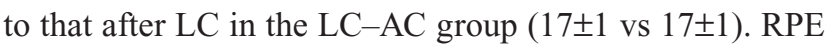
after the training was also similar between the two groups ( $18 \pm 1$ in the LC group vs $17 \pm 1$ in the LC-AC group). Since the workload and the number of sets were progressively increased throughout the 16-week training period, RPE was unchanged throughout the intervention. Blood lactate level was also similar between the two groups $(12 \pm 1 \mathrm{mM}$ for LC group and $11 \pm 1 \mathrm{mM}$ for LC-AC group).

\section{Incremental exercise tests}

As shown in Table 2, increases in peak $\mathrm{W}$ from baseline in the LC incremental exercise test were observed in both groups. The increase in peak $\mathrm{W}$ was $>10 \%$ in the LC group, except for one participant (L7) who could not finish the 16-week HIIT because of his aggravating migraine attacks near the end of the 16-week intervention. In fact, the increase in peak $\mathrm{W}$ in the LC group, except for that participant (L7), was greater than that in the LC-AC group $(28 \% \pm 10 \%$ vs $11 \% \pm 9 \%, P<0.05)$. Furthermore, an increase in $\mathrm{VO}_{2}$ peak was found only in the $\mathrm{LC}$ group. In the $\mathrm{AC}-\mathrm{LC}$ group, an increase in $\mathrm{VO}_{2}$ peak was not observed in one participant (A2) due to a robust weight gain as a result of changing his diet behavior because of increased opportunities for dinner parties. There were increases in $\mathrm{O}_{2}$ pulse, which is an index for stroke volume, in both groups. 
Table 2 Results of leg-cycling incremental exercise tests

\begin{tabular}{|c|c|c|c|c|c|c|c|c|c|c|c|c|}
\hline \multirow[t]{2}{*}{ Participant } & \multicolumn{3}{|c|}{$\mathrm{VO}_{2}$ peak $\left(\mathrm{mL} \cdot \mathrm{min}^{-1} \cdot \mathrm{kg}^{-1}\right)$} & \multicolumn{3}{|c|}{ Watt peak (W) } & \multicolumn{3}{|c|}{ HR peak (beat $\cdot \min$ ) } & \multicolumn{3}{|c|}{$\mathrm{O}_{2}$ pulse $\left(\mathrm{mL} \cdot\right.$ beat $\left.^{-1}\right)$} \\
\hline & Pre & Post & $\%$ & Pre & Post & $\%$ & Pre & Post & $\%$ & Pre & Post & $\%$ \\
\hline \multicolumn{13}{|l|}{ LC } \\
\hline LI & 37.9 & 44.6 & 17.7 & 200 & 255 & 27.5 & 173 & 180 & 4.0 & 17.8 & 20.0 & 12.4 \\
\hline L2 & 33.0 & 43.0 & 30.3 & 173 & 240 & 38.7 & 177 & 178 & 0.6 & 13.3 & 16.9 & 27.4 \\
\hline L3 & 41.6 & 51.3 & 23.3 & 218 & 270 & 23.9 & 180 & 185 & 2.8 & 14.0 & 16.5 & 18.2 \\
\hline L4 & 39.5 & 50.0 & 26.6 & 215 & 300 & 39.5 & 172 & 188 & 9.3 & 17.7 & 21.1 & 19.1 \\
\hline L5 & 35.8 & 43.5 & 21.5 & 210 & 270 & 28.6 & 176 & $|8|$ & 2.8 & 15.5 & 17.4 & 12.9 \\
\hline L6 & 48.0 & 55.0 & 14.6 & 230 & 255 & 10.9 & 186 & 185 & -0.5 & 17.5 & 20.5 & 17.1 \\
\hline L7 & 52.0 & 51.0 & -1.9 & 270 & 280 & 3.7 & 184 & 184 & 0.0 & 20.3 & 20.2 & -0.4 \\
\hline Mean & 41.1 & $48.3^{*}$ & 17.6 & 217 & $267^{*}$ & 23.4 & 178 & 183 & 2.6 & 16.6 & 18.9* & 13.9 \\
\hline SD & 6.2 & 4.3 & 9.8 & 27 & 18 & 12.4 & 5 & 3 & 3.1 & 2.5 & 1.9 & 8.5 \\
\hline \multicolumn{13}{|l|}{ LC-AC } \\
\hline Al & 44.9 & 50.3 & 12.0 & 255 & 260 & 2.0 & 188 & 180 & -4.3 & 18.0 & 21.0 & 16.4 \\
\hline $\mathrm{A} 2$ & 40.6 & 40.0 & -1.5 & 263 & 278 & 5.7 & 197 & 198 & 0.5 & 18.1 & 18.8 & 4.0 \\
\hline $\mathrm{A} 3$ & 38.0 & 44.0 & 15.8 & 225 & 265 & 17.8 & 155 & 157 & 1.3 & 18.8 & 21.4 & 13.9 \\
\hline $\mathrm{A} 4$ & 31.4 & 41.0 & 30.6 & 180 & 230 & 27.8 & 165 & 167 & 1.2 & 13.6 & 17.1 & 25.9 \\
\hline A5 & 37.2 & 39.4 & 5.9 & 200 & 215 & 7.5 & 172 & 155 & -9.9 & 16.3 & 19.3 & 18.2 \\
\hline Mean & 38.4 & 42.9 & 11.8 & 225 & $250 *$ & 11 & 175 & $17 \mid$ & -2.3 & 17.0 & $19.5^{*}$ & 14.7 \\
\hline SD & 4.4 & 4.0 & 10.7 & 32 & 23 & 9.4 & 15 & 16 & 4.3 & 2.1 & 1.7 & 7.9 \\
\hline
\end{tabular}

Notes: $* P<0.05$, by Wilcoxon rank test. Pre and post refer to the 16-week high-intensity interval training program.

Abbreviations: HR, heart rate; LC, leg-cycling group; LC-AC, leg-cycling and arm-cranking group; SD, standard deviation; VO ${ }_{2}$, oxygen uptake that can be utilized one minute.

As shown in Table 3, an increase in peak $\mathrm{W}$ from baseline in the AC incremental exercise test was observed only in the $\mathrm{LC}-\mathrm{AC}$ group, which was $>10 \%$ in all the participants (A1-A5) in the LC-AC group. On the other hand, there was no increase in peak $\mathrm{W}$ in the $\mathrm{LC}$ group. One participant in

Table 3 Results of arm-cranking incremental exercise tests

\begin{tabular}{|c|c|c|c|c|c|c|}
\hline \multirow[t]{2}{*}{ Participant } & \multicolumn{3}{|c|}{ Watt peak (W) } & \multicolumn{3}{|c|}{ HR peak (beat $\cdot \mathrm{min}^{-1}$ ) } \\
\hline & Pre & Post & $\%$ & Pre & Post & $\%$ \\
\hline \multicolumn{7}{|l|}{ LC } \\
\hline LI & 95 & 100 & 5.3 & 151 & 158 & 4.6 \\
\hline L2 & 75 & 65 & -13.3 & 165 & 152 & -7.9 \\
\hline L3 & 90 & 75 & -16.7 & 170 & 145 & -14.7 \\
\hline L4 & 115 & 125 & 8.7 & 160 & 175 & 9.4 \\
\hline L5 & 90 & 90 & 0.0 & 161 & 166 & 3.1 \\
\hline L6 & 95 & 110 & 15.8 & 180 & $|7|$ & -5.0 \\
\hline L7 & 110 & NM & NM & 175 & NM & NM \\
\hline Mean & 93 & 94 & 0.9 & 165 & 161 & -2.0 \\
\hline SD & 12 & 20 & 12.1 & 9 & II & 9.0 \\
\hline \multicolumn{7}{|l|}{ LC-AC } \\
\hline Al & 115 & 130 & 13.0 & 175 & 180 & 2.9 \\
\hline $\mathrm{A} 2$ & 100 & 130 & 30.0 & 184 & 191 & 3.8 \\
\hline $\mathrm{A} 3$ & 90 & 115 & 27.8 & 147 & 157 & 6.8 \\
\hline A4 & 80 & 110 & 37.5 & 150 & 147 & -2.0 \\
\hline A5 & 95 & 115 & 21.1 & 164 & 142 & -13.4 \\
\hline Mean & 96 & $120^{*}$ & 25.0 & 164 & 163 & -0.4 \\
\hline SD & 12 & 8 & 8.3 & 14 & 19 & 7.1 \\
\hline
\end{tabular}

Notes: $* P<0.05$, by Wilcoxon rank test. Pre and post refer to the 16-week highintensity interval training program.

Abbreviations: HR, heart rate; LC, leg-cycling group; LC-AC, leg-cycling and armcranking group; NM, not measured; SD, standard deviation. the LC group (L7) was unable to complete the posttest due to headache aura.

\section{Morphological characteristics}

Total LBM, including leg LBM, was not increased from baseline in the LC group, while four participants (L1, L2, L3, and L5) gained $\geq 2.5 \%$ of LBM without body weight gain (Table 4). There was no increase in LBM in the LC-AC group, either. Using MRI, the CSAs of trunk and thigh muscles were examined before and after the 16-week LC- and LC-AC-HIIT programs (Table 5 and Figure 2A-D). Both groups displayed increases in the CSA of quadriceps femoris muscles (both $P<0.05$, Table 5). Additionally, increases from baseline were observed in $\mathrm{m}$ psoas major and $\mathrm{m}$ anterolateral abdominal in the LC-AC group (both $P<0.05$ ).

There was a positive association between percent change in $\mathrm{VO}_{2}$ peak and the CSA of $\mathrm{m}$ psoas major in each group ( $\mathrm{LC}$ group, $\rho=0.89, P<0.01$; LC - AC group, $\rho=0.90, P<0.05$ ). On the other hand, no correlation was observed between percent changes in $\mathrm{VO}_{2}$ peak and the CSA of quadriceps femoris muscles.

\section{Discussion}

In the present preliminary study, we hypothesized that a combined 16-week LC- and AC-HIIT program consisting of 16- to 24-minute twice-weekly sessions would improve maximum workload and $\mathrm{VO}_{2}$ peak and lead to morphological changes 
Table 4 Effects of high-intensity interval training on body composition

\begin{tabular}{|c|c|c|c|c|c|c|c|c|c|c|c|c|c|c|c|}
\hline & \multicolumn{3}{|c|}{ BW (kg) } & \multirow{2}{*}{\multicolumn{3}{|c|}{$\begin{array}{l}\text { Total body } \\
\text { BFLBM (kg) }\end{array}$}} & \multirow{2}{*}{\multicolumn{3}{|c|}{$\begin{array}{l}\text { Upper-body } \\
\text { BFLBM (kg) }\end{array}$}} & \multirow{2}{*}{\multicolumn{3}{|c|}{$\begin{array}{l}\text { Trunk } \\
\text { BFLBM (kg) }\end{array}$}} & \multirow{2}{*}{\multicolumn{3}{|c|}{$\begin{array}{l}\text { Lower-body } \\
\text { BFLBM (kg) }\end{array}$}} \\
\hline & \multirow[t]{2}{*}{ Pre } & \multirow[t]{2}{*}{ Post } & \multirow[t]{2}{*}{$\%$} & & & & & & & & & & & & \\
\hline & & & & Pre & Post & $\%$ & Pre & Post & $\%$ & Pre & Post & $\%$ & Pre & Post & $\%$ \\
\hline \multicolumn{16}{|l|}{ LC } \\
\hline LI & 81.2 & 80.7 & -0.6 & 57.0 & 59.0 & 3.5 & 6.0 & 5.8 & -3.7 & 27.1 & 29.3 & 8.1 & 19.6 & 19.5 & -0.2 \\
\hline L2 & 71.2 & 70.0 & -1.7 & 47.4 & 48.9 & 3.1 & 4.3 & 4.3 & 1.0 & 20.9 & 22.5 & 8.0 & 17.8 & 17.7 & -1.0 \\
\hline L3 & 60.4 & 59.5 & -1.5 & 46.2 & 48.1 & 4.2 & 5.1 & 5.2 & 2.2 & 21.5 & 22.5 & 4.6 & 15.5 & 16.3 & 5.2 \\
\hline L4 & 77.0 & 79.2 & 2.9 & 54.6 & 55.6 & 1.8 & 6.0 & 5.6 & -6.0 & 25.1 & 25.7 & 2.4 & 19.1 & 20.1 & 4.8 \\
\hline L5 & 76.0 & 72.6 & -4.5 & 54.9 & 56.3 & 2.5 & 7.0 & 7.0 & 0.1 & 25.0 & 25.9 & 3.8 & 18.4 & 18.9 & 2.3 \\
\hline L6 & 67.9 & 69.0 & 1.6 & 52.3 & 51.9 & -0.8 & 5.4 & 6.1 & 14.3 & 25.7 & 24.2 & -5.7 & 17.0 & 17.6 & 3.3 \\
\hline L7 & 71.8 & 72.9 & 1.5 & 59.2 & 58.6 & -1.0 & 6.0 & 6.1 & 2.3 & 29.7 & 27.9 & -6.1 & 18.9 & 20.0 & 5.8 \\
\hline Mean & 72.2 & 72.0 & -0.3 & 53.1 & 54.1 & 1.8 & 5.7 & 5.7 & 1.2 & 25.0 & 25.4 & 1.8 & 18.1 & 18.6 & 2.8 \\
\hline $\mathrm{SD}$ & 6.3 & 6.5 & 2.3 & 4.4 & 4.1 & 1.9 & 0.8 & 0.8 & 6.0 & 2.8 & 2.4 & 5.5 & 1.3 & 1.3 & 2.5 \\
\hline \multicolumn{16}{|l|}{ LC-AC } \\
\hline Al & 75.4 & 75.0 & -0.5 & 59.3 & 59.6 & 0.6 & 6.4 & 6.5 & 1.2 & 27.3 & 27.3 & -0.1 & 21.1 & 21.2 & 0.5 \\
\hline $\mathrm{A} 2$ & 87.6 & 92.9 & 6.1 & 58.8 & 59.5 & 1.2 & 7.6 & 7.4 & -2.9 & 25.2 & 26.8 & 6.4 & 21.6 & 21.0 & -3.2 \\
\hline $\mathrm{A} 3$ & 76.5 & 76.2 & -0.4 & 54.8 & 58.4 & 6.4 & 6.1 & 6.3 & 4.3 & 24.6 & 27.7 & 12.2 & 19.7 & 19.9 & 1.0 \\
\hline A4 & 71.5 & 69.8 & -2.4 & 52.9 & 51.8 & -1.9 & 5.6 & 5.8 & 3.2 & 23.9 & 23.0 & -3.9 & 19.3 & 18.8 & -2.4 \\
\hline A5 & 75.5 & 75.9 & 0.5 & 46.3 & 48.1 & 4.0 & 5.7 & 6.0 & 4.4 & 19.3 & 20.7 & 7.4 & 17.1 & 17.1 & 0.2 \\
\hline Mean & 77.3 & 78.0 & 0.9 & 54.4 & 55.5 & 2.0 & 6.3 & 6.4 & 1.7 & 24.1 & 25.1 & 4.2 & 19.8 & 19.6 & -0.8 \\
\hline SD & 5.4 & 7.8 & 2.9 & 4.7 & 4.7 & 2.9 & 0.7 & 0.5 & 2.7 & 2.7 & 2.8 & 5.7 & 1.6 & 1.5 & 1.7 \\
\hline
\end{tabular}

Note: Pre and post refer to the 16-week high-intensity interval training program.

Abbreviations: BFLBM, bone-free lean body mass; BW, body weight; LC, leg-cycling group; LC-AC, leg-cycling and arm-cranking group; SD, standard deviation.

in healthy men equal to an LC-HIIT program of identical training intensity and volume in healthy men. Increases from baseline in peak $\mathrm{W}$ during $\mathrm{LC}$ were detected in both groups (LC group, $+23 \%$; LC-AC group, $+11 \%$ ). Increases from baseline in the CSA of quadriceps femoris muscles were also observed in both groups (LC group, $+11 \%$; LC-AC group, $+5 \%$ ). Moreover, muscle hypertrophy in the $\mathrm{m}$ psoas major and $\mathrm{m}$ anterolateral abdominal, as well as significant improvements in $\mathrm{AC}$ aerobic capacity, were observed in the LC-AC group. Interestingly, improvement of aerobic fitness was associated with muscle hypertrophy in $\mathrm{m}$ psoas major, instead of that in quadriceps femoris muscles. Our results suggest that a 16 -week LC-AC-HIIT program can induce improvements in both upper- and lower-body aerobic

Table 5 Effects of high-intensity interval training on cross-sectional area of trunk and thigh muscles

\begin{tabular}{|c|c|c|c|c|c|c|c|c|c|c|c|c|c|c|c|}
\hline & \multirow{2}{*}{\multicolumn{3}{|c|}{$\begin{array}{l}\text { M psoas } \\
\text { major }\left(\mathrm{cm}^{2}\right)\end{array}$}} & \multirow{2}{*}{\multicolumn{3}{|c|}{$\begin{array}{l}\text { M anterolateral } \\
\text { abdominal }\left(\mathrm{cm}^{2}\right)\end{array}$}} & \multirow{2}{*}{\multicolumn{3}{|c|}{$\begin{array}{l}\text { Spinal } \\
\text { muscle }\left(\mathrm{cm}^{2}\right)\end{array}$}} & \multirow{2}{*}{\multicolumn{3}{|c|}{$\begin{array}{l}\text { Quadriceps } \\
\text { femoris }\left(\mathrm{cm}^{2}\right)\end{array}$}} & \multicolumn{3}{|c|}{ Hamstrings $\left(\mathrm{cm}^{2}\right)$} \\
\hline & & & & & & & & & & & & & \multirow[t]{2}{*}{ Pre } & \multirow[t]{2}{*}{ Post } & \multirow[t]{2}{*}{$\%$} \\
\hline & Pre & Post & $\%$ & Pre & Post & $\%$ & Pre & Post & $\%$ & Pre & Post & $\%$ & & & \\
\hline \multicolumn{16}{|l|}{ LC } \\
\hline LI & 28.2 & 27.5 & -2.5 & 43.8 & 51.0 & 16.7 & 48.2 & 51.2 & 6.2 & 80.0 & 90.6 & 13.2 & 84.2 & 86.9 & 3.1 \\
\hline L2 & 22.7 & 25.2 & 10.7 & 39.0 & 37.5 & -3.8 & 36.3 & 37.7 & 3.7 & 54.9 & 59.5 & 8.3 & 67.2 & 66.7 & -0.7 \\
\hline L3 & 21.9 & 21.9 & 0.3 & 38.9 & 41.7 & 7.3 & 41.5 & 41.2 & -0.7 & 54.2 & 60.6 & 11.8 & 51.6 & 54.5 & 5.6 \\
\hline L4 & 19.9 & 23.8 & 19.3 & 48.0 & 51.5 & 7.4 & 51.7 & 52.1 & 0.8 & 71.2 & 77.5 & 8.8 & 69.5 & 74.2 & 6.9 \\
\hline L5 & 21.3 & 22.5 & 5.6 & 37.1 & 35.6 & -4.2 & 43.8 & 39.0 & -10.8 & 63.5 & 66.1 & 4.1 & 62.0 & 60.7 & -2.0 \\
\hline L6 & 31.7 & 27.4 & -13.6 & 40.4 & 41.4 & 2.3 & 44.8 & 47.0 & 5.0 & 65.0 & 66.2 & 1.9 & 64.6 & 68.3 & 5.7 \\
\hline L7 & 26.7 & 25.2 & -5.5 & 48.5 & 50.3 & 3.6 & 47.7 & 46.0 & -3.7 & 64.8 & 70.9 & 9.3 & 69.0 & 72.5 & 5.1 \\
\hline Mean & 29.6 & 29.8 & 0.5 & 47.2 & 49.2 & 4.0 & 49.8 & 49.9 & 0.1 & 69.8 & $75.2^{*}$ & 7.7 & 71.9 & 74.1 & 3.1 \\
\hline SD & 4.0 & 2.0 & 10.1 & 4.2 & 6.2 & 6.7 & 4.7 & 5.3 & 5.9 & 8.3 & 10.1 & 3.7 & 9.1 & 9.6 & 3.2 \\
\hline \multicolumn{16}{|l|}{ LC-AC } \\
\hline Al & 31.3 & 31.6 & 0.9 & 36.8 & 40.2 & 9.3 & 47.5 & 44.0 & -7.5 & 72.8 & 76.1 & 4.5 & 64.4 & 75.8 & 17.7 \\
\hline $\mathrm{A} 2$ & 25.5 & 25.6 & 0.5 & 50.1 & 52.8 & 5.5 & 44.0 & 52.8 & 19.9 & 80.5 & 88.2 & 9.6 & 73.1 & 77.4 & 5.9 \\
\hline $\mathrm{A} 3$ & 26.0 & 32.6 & 25.3 & 31.1 & 35.5 & 14.1 & 41.7 & 39.9 & -4.2 & 67.0 & 70.0 & 4.4 & 64.5 & 62.3 & -3.3 \\
\hline A4 & 22.7 & 28.5 & 25.5 & 31.8 & 33.6 & 5.6 & 39.0 & 35.1 & -10.1 & 66.2 & 68.4 & 3.3 & 62.4 & 43.0 & -31.1 \\
\hline A5 & 20.5 & 21.9 & 6.7 & 37.4 & 38.8 & 3.6 & 37.6 & 41.8 & 10.9 & 65.4 & 68.9 & 5.3 & 69.1 & 65.3 & -5.5 \\
\hline Mean & 30.2 & $33.0 *$ & 9.4 & 42.4 & $45.2^{*}$ & 6.5 & 47.0 & 47.7 & 1.5 & 75.4 & $79.3^{*}$ & 5.2 & 71.7 & 69.8 & -2.7 \\
\hline SD & 3.6 & 3.9 & 11.3 & 6.8 & 6.7 & 3.7 & 3.5 & 5.8 & 11.6 & 5.7 & 7.5 & 2.2 & 3.9 & 12.3 & 16.1 \\
\hline
\end{tabular}

Notes: $* P<0.05$, by Wilcoxon rank test. Pre and post refer to the 16-week high-intensity interval training program.

Abbreviations: LC, leg-cycling group; LC-AC, leg-cycling and arm-cranking group; m, musculus; SD, standard deviation. 

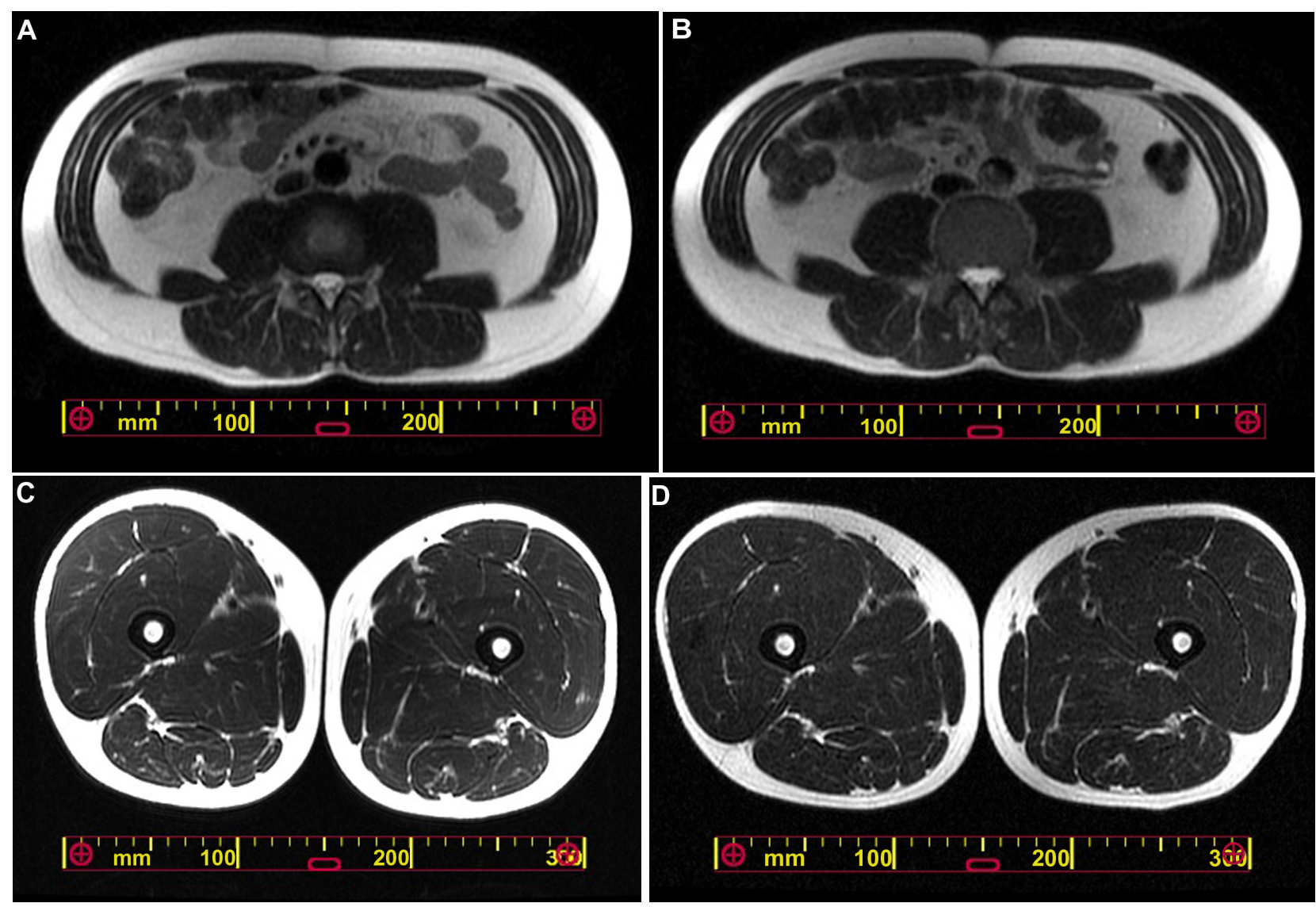

Figure 2 Representative axial magnetic resonance imaging scans of the L3-L4 disc space level and the half-way point of the femur level at pretraining (A and C) and posttraining (B and $\mathbf{D})$.

capacity and promote muscle hypertrophy of $\mathrm{m}$ psoas major and $\mathrm{m}$ anterolateral abdominal as well as quadriceps femoris muscles.

In LC incremental exercise tests, peak $\mathrm{W}$ significantly improved in both groups, while an increase in $\mathrm{VO}_{2}$ peak was observed only in the LC group. Ten sets of LC-HIIT per session, as in the recent reports, ${ }^{23,26}$ induced $>10 \%$ of increases in peak $\mathrm{W}$ in six of seven participants and $>20 \%$ of that in five of seven participants, while five sets of LC-HIIT per session induced $>10 \%$ of increases in peak $\mathrm{W}$ in two of five participants and $>20 \%$ of that in only one of five participants. A recent meta-analysis showed that an improvement in the $\mathrm{VO}_{2}$ peak was $\sim 20 \%$ after HIIT, consistent with the current study in the LC group, while it was $\sim 10 \%$ after moderateintensity continuous training. ${ }^{27}$ It has also been reported that transfer effects of endurance training (ie, increase of performance measured during exercise with untrained muscle) is more controversial ${ }^{28}$ than specific training (ie, performance measured during exercise with trained muscles). In arm training studies, an increase of $>30 \%$ of $\mathrm{VO}_{2}$ peak (measured in arm test) is necessary to obtain a significant transfer effect, while no transfer effects are observed where the $\mathrm{VO}_{2}$ peak improvement is $<20 \% .{ }^{29}$ In the current study, though there was a $25 \%$ increase in peak $\mathrm{W}$ during the $\mathrm{AC}$ incremental test in the LC-AC group, it appears that peripheral adaptation (ie, greater muscle endurance capacity in the lower extremities) as transfer effects did not occur, while $>10 \%$ increases in $\mathrm{O}_{2}$ pulse, an index of stroke volume, were observed in both groups, implying that central (ie, cardiac) adaptation did occur similarly between the two groups. There were no transfer effects for AC aerobic capacity in the LC group either, despite $\sim 20 \%$ increase in $\mathrm{VO}_{2}$ peak measured in an LC incremental test.

The observation that the HIIT program increased the CSA of quadriceps femoris muscles is supported by Boudou et al, ${ }^{19}$ who reported that an 8-week LC exercise program comprising continuous endurance and HIIT exercises led to a $24 \%$ increase in the CSA of these muscles, though the increase was much greater compared with the current study. This can be partly explained by the characteristics of participants, who were older diabetics and were likely to be less fit and less physically active, as well as the difference in exercise protocol. 
Trunk muscles are activated in response to dynamic flexion of the torso, trunk rotation movement, and cycle ergometer exercise. ${ }^{30-32}$ Trunk muscle activation would also be required to stabilize the trunk for the $\mathrm{AC}$ and $\mathrm{LC}$ exercises. In fact, muscle hypertrophy was observed in the $\mathrm{m}$ psoas major and $\mathrm{m}$ anterolateral abdominal in the $\mathrm{LC}-\mathrm{AC}$ group. Moreover, improvement of aerobic fitness was associated with muscle hypertrophy in $\mathrm{m}$ psoas major, instead of that in quadriceps femoris muscles, not only in the LC-AC group but also in the LC group. A recent report using positron emission tomography to evaluate individual skeletal muscle activity during pedaling exercise showed robust increased activity of iliopsoas muscle, especially iliacus muscle, as well as of muscles of the anterior part of the thigh. Unfortunately, iliacus muscle was not measured in the current study. However, it is likely that participants who could use iliopsoas muscle more effectively could perform better cycling performance with higher oxygen uptake due to more muscle working. In fact, it has been reported that resistance training of the arms, chest, and trunk improved cycling exercise capacity. ${ }^{33}$

Maximal $\mathrm{VO}_{2}$ during arm work in untrained subjects has been reported as $\sim 70 \%$ of maximal $\mathrm{VO}_{2}$ during leg work, ${ }^{34}$ since upper-body exercises involve a small muscular mass and oxidative machinery in untrained arms may be less developed. ${ }^{34}$ Moreover, at any given $\mathrm{VO}_{2}$, $\mathrm{HR}$ is approximately $20 \%$ higher during arm work than during leg exercise, resulting in higher RPE during arm work. ${ }^{25}$ Therefore, there was a concern that $\mathrm{AC}$ might not be suitable for substituting the LC. In the current study, AC-HIIT was well tolerated and RPE was similar to that in LC-HIIT. Moreover, there was $>10 \%$ increases in $\mathrm{O}_{2}$ pulse, an index of stroke volume, in both groups, suggesting that central (ie, cardiac) adaptation did occur similarly between the two groups.

Our results suggest that a combination of LC- and ACHIIT can induce improvements in both upper- and lower-body endurance aerobic capacity and promote muscle hypertrophy of $\mathrm{m}$ psoas major and $\mathrm{m}$ anterolateral abdominal as well as quadriceps femoris muscles. A 16-week LC-AC-HIIT program may induce an increase in the size of $\mathrm{m}$ psoas major, which can be related to LC exercise capacity.

Several limitations of this study should be considered when interpreting and generalizing the findings presented here. First, the study consisted of men with a relatively wide age range. Second, the study was underpowered due to the small sample size resulting from budget and facility constraints, which may be reflected in the failure to detect differences in the selected measurement outcomes. Finally, although participants performed the HIIT programs with a high RPE during the last set of each training session and under supervision, the volume of training, which ranged from eight to 12 sets per session, varied among participants due to differences in their physical condition.

\section{Conclusion}

In conclusion, we have demonstrated that a 16-week LC-ACHIIT program can induce improvements in both upper- and lower-body exercise tolerance capacities, accompanied with muscle hypertrophy of $\mathrm{m}$ psoas major and $\mathrm{m}$ anterolateral abdominal as well as quadriceps femoris muscles, in healthy men.

\section{Acknowledgments}

We thank Yoshinobu Nunokawa, Yasunari Hisashi, Koshi Okabe, Yasutomi Shimada, Yasuko Kamitaki, and Yujiro Nakamura and the staff of the Radiology Department for their support with the MRI and DXA measurements. We are also indebted to the staff of the Institute for Integrated Sports Medicine for their assistance with data collection and maintenance of training and evaluation equipment. Most importantly, we express our appreciation to the research volunteers who participated in this study. The present study was financially supported by the Nateglinide Memorial Toyoshima Research and Education Fund (2011) and a Grant-in-Aid for Young Scientists (B; No 22700700).

\section{Disclosure}

The authors report no conflicts of interest in this work.

\section{References}

1. Little JP, Gillen JB, Percival ME, et al. Low-volume high-intensity interval training reduces hyperglycemia and increases muscle mitochondrial capacity in patients with type 2 diabetes. J Appl Physiol. 2011;111(6):1554-1560.

2. Nilsson BB, Westheim A, Risberg MA. Long-term effects of a groupbased high-intensity aerobic interval-training program in patients with chronic heart failure. Am J Cardiol. 2008;102(9):1220-1224.

3. Rognmo O, Hetland E, Helgerud J, Hoff J, Slordahl SA. High intensity aerobic interval exercise is superior to moderate intensity exercise for increasing aerobic capacity in patients with coronary artery disease. Eur J Cardiovasc Prev Rehabil. 2004;11(3):216-222.

4. Tjonna AE, Lee SJ, Rognmo O, et al. Aerobic interval training versus continuous moderate exercise as a treatment for the metabolic syndrome: a pilot study. Circulation. 2008;118(4):346-354.

5. Warburton DE, McKenzie DC, Haykowsky MJ, et al. Effectiveness of high-intensity interval training for the rehabilitation of patients with coronary artery disease. Am J Cardiol. 2005;95(9):1080-1084.

6. Wisloff U, Stoylen A, Loennechen JP, et al. Superior cardiovascular effect of aerobic interval training versus moderate continuous training in heart failure patients: a randomized study. Circulation. 2007;115(24):3086-3094.

7. Burgomaster KA, Heigenhauser GJ, Gibala MJ. Effect of shortterm sprint interval training on human skeletal muscle carbohydrate metabolism during exercise and time-trial performance. J Appl Physiol. 2006;100(6):2041-2047. 
8. Burgomaster KA, Hughes SC, Heigenhauser GJ, Bradwell SN, Gibala MJ Six sessions of sprint interval training increases muscle oxidative potential and cycle endurance capacity in humans. J Appl Physiol. 2005;98(6):1985-1990.

9. Carroll TJ, Herbert RD, Munn J, Lee M, Gandevia SC. Contralateral effects of unilateral strength training: evidence and possible mechanisms. J Appl Physiol. 2006;101(5):1514-1522.

10. Rakobowchuk M, Tanguay S, Burgomaster KA, Howarth KR, Gibala MJ, MacDonald MJ. Sprint interval and traditional endurance training induce similar improvements in peripheral arterial stiffness and flowmediated dilation in healthy humans. Am J Physiol Regul Integr Comp Physiol. 2008;295(1):R236-R242.

11. Bartlett JD, Close GL, MacLaren DP, Gregson W, Drust B, Morton JP. High-intensity interval running is perceived to be more enjoyable than moderate-intensity continuous exercise: implications for exercise adherence. J Sports Sci. 2011;29(6):547-553.

12. Oliveira BR, Slama FA, Deslandes AC, Furtado ES, Santos TM Continuous and high-intensity interval training: which promotes higher pleasure? PLoS One. 2013;8(11):e79965.

13. Gibala MJ, Little JP, Macdonald MJ, Hawley JA. Physiological adaptations to low-volume, high-intensity interval training in health and disease. J Physiol. 2012;590(Pt 5):1077-1084.

14. Gosselin LE, Kozlowski KF, DeVinney-Boymel L, Hambridge C. Metabolic response of different high-intensity aerobic interval exercise protocols. J Strength Cond Res. 2012;26(10):2866-2871.

15. Hendy AM, Spittle M, Kidgell DJ. Cross education and immobilisation: mechanisms and implications for injury rehabilitation. J Sci Med Sport 2012;15(2):94-101.

16. Tanaka H. Effects of cross-training. Transfer of training effects on VO2max between cycling, running and swimming. Sports Med. 1994;18(5):330-339.

17. Harber MP, Gallagher PM, Creer AR, Minchev KM, Trappe SW. Single muscle fiber contractile properties during a competitive season in male runners. Am J Physiol Regul Integr Comp Physiol. 2004;287(5):R1124-R1131.

18. Kohn TA, Essen-Gustavsson B, Myburgh KH. Specific muscle adaptations in type II fibers after high-intensity interval training of well-trained runners. Scand J Med Sci Sports. 2011;21(6):765-772.

19. Boudou P, Sobngwi E, Mauvais-Jarvis F, Vexiau P, Gautier JF. Absence of exercise-induced variations in adiponectin levels despite decreased abdominal adiposity and improved insulin sensitivity in type 2 diabetic men. Eur J Endocrinol. 2003;149(5):421-424.

20. McPhee JS, Williams AG, Degens H, Jones DA. Inter-individual variability in adaptation of the leg muscles following a standardised endurance training programme in young women. Eur J Appl Physiol. 2010;109(6):1111-1118.
21. Sale DG. Neural adaptation to resistance training. Med Sci Sports Exerc. 1988;20(Suppl 5):S135-S145.

22. Abe T, DeHoyos DV, Pollock ML, Garzarella L. Time course for strength and muscle thickness changes following upper and lower body resistance training in men and women. Eur J Appl Physiol. 2000;81(3):174-180.

23. Little JP, Safdar A, Wilkin GP, Tarnopolsky MA, Gibala MJ. A practical model of low-volume high-intensity interval training induces mitochondrial biogenesis in human skeletal muscle: potential mechanisms. J Physiol. 2010;588(Pt 6):1011-1022.

24. Borg GA. Psychophysical bases of perceived exertion. Med Sci Sports Exerc. 1982;14(5):377-381.

25. Olivier N, Legrand R, Rogez J, Berthoin S, Prieur F, Weissland T. One-leg cycling versus arm cranking: which is most appropriate for physical conditioning after knee surgery? Arch Phys Med Rehabil. 2008;89(3):508-512.

26. Hood MS, Little JP, Tarnopolsky MA, Myslik F, Gibala MJ. Low-volume interval training improves muscle oxidative capacity in sedentary adults. Med Sci Sports Exerc. 2011;43(10):1849-1856

27. Weston KS, Wisloff U, Coombes JS. High-intensity interval training in patients with lifestyle-induced cardiometabolic disease: a systematic review and meta-analysis. Br J Sports Med. 2014;48(16): 1227-1234.

28. Franklin BA. Aerobic exercise training programs for the upper body. Med Sci Sports Exerc. 1989;21(Suppl 5):S141-S148.

29. Tordi N, Belli A, Mougin F, Rouillon JD, Gimenez M. Specific and transfer effects induced by arm or leg training. Int J Sports Med. 2001;22(7):517-524.

30. Juker D, McGill S, Kropf P, Steffen T. Quantitative intramuscular myoelectric activity of lumbar portions of psoas and the abdominal wall during a wide variety of tasks. Med Sci Sports Exerc. 1998;30(2): 301-310.

31. Gondoh Y, Tashiro M, Itoh M, et al. Evaluation of individual skeletal muscle activity by glucose uptake during pedaling exercise at different workloads using positron emission tomography. J Appl Physiol. 2009;107(2):599-604.

32. Andersson E, Oddsson L, Grundstrom H, Thorstensson A. The role of the psoas and iliacus muscles for stability and movement of the lumbar spine, pelvis and hip. Scand J Med Sci Sports. 1995;5(1):10-16.

33. Segerstrom AB, Holmback AM, Hansson O, et al. Relation between cycling exercise capacity, fiber-type composition, and lower extremity muscle strength and muscle endurance. J Strength Cond Res. 2011;25(1):16-22.

34. Pendergast DR. Cardiovascular, respiratory, and metabolic responses to upper body exercise. Med Sci Sports Exerc. 1989; 21(Suppl 5):S121-S125.
Open Access Journal of Sports Medicine

\section{Publish your work in this journal}

Open Access Journal of Sports Medicine is an international, peer-reviewed, open access journal publishing original research, reports, reviews and commentaries on all areas of sports medicine. The manuscript management system is completely online and includes a very quick and fair peer-review system.

\section{Dovepress}

Visit http://www.dovepress.com/testimonials.php to read real quotes from published authors. 